\title{
An Alternative Algorithm for a Sliding Window ULV Decomposition*
}

\author{
H. Erbay, Kırıkkale, and J. Barlow, State College
}

February 25, 2005

\begin{abstract}
The ULV decomposition (ULVD) is an important member of a class of rank-revealing two-sided orthogonal decompositions used to approximate the singular value decomposition (SVD). The ULVD can be modified much faster than the SVD.

When modifiying the ULVD, the accurate computation of the subspaces is required in certain time varying applications in signal processing. In this paper we present an updating algorithm which is suitable for large scaled matrices of low rank and as effective as alternatives. The algorithm runs in $O\left(n^{2}\right)$ time.
\end{abstract}

AMS Subject Classification: 65F05, 65F30, 65F50

Key words: Modifying decompositions, norm and condition estimation, subspaces.

\section{Introduction}

In the exponential window method [13], a new data $\mathbf{x}(t+1) \in \mathbf{R}^{n}$ is continuously added to the data matrix $X(t) \in \mathbf{R}^{\left(m_{0}+t\right) \times n}$ with $m_{0} \geq n$ multiplied by a positive forgetting factor $\alpha<1, t=0,1,2, \ldots$ where for each $t$

$$
X(t)=\left(\begin{array}{c}
\mathbf{x}(t)^{T} \\
\alpha \mathbf{x}(t-1)^{T} \\
\alpha^{2} \mathbf{x}(t-2)^{T} \\
\vdots \\
\alpha^{t} X(0)
\end{array}\right)
$$

where $X(0) \in \mathbf{R}^{m_{0} \times n}$ is an initial matrix. Thus it is desirable to obtain SVD of the matrix

$$
X(t+1)=\left(\begin{array}{c}
\mathbf{x}(t+1)^{T} \\
\alpha X(t)
\end{array}\right)
$$

${ }^{*}$ Research fund by the National Science Foundation under contract no. CCR-9732081 
We might consider using the SVD of $X(t)$ to compute that of $X(t+1)$, but "practical" procedures for that require $O\left(n^{3}\right)$ flops. The adaptive fast multipole procedure of $\mathrm{Gu}$ and Eisenstat [8] requires $O\left(n^{2}\right)$ flops, but no robust software is available for it, and its flop count is $c n^{2}+o\left(n^{2}\right)$ where $c$ is a very large constant that depends upon machine precision.

Thus, we would like an approximation to the SVD of $X(t+1)$ which can be computed from a similar approximation for $X(t)$ in significantly fewer operations. The ULV decomposition (ULVD) is such an approximation.

Throughout the paper $\|\cdot\|$ will denote the two norm, and $\|\cdot\|_{F}$ will denote the Frobenius norm.

The ULVD is a special case of the two-sided orthogonal decompositions defined by Faddeev, Kublanovskaya, and Faddeeva [5] and Hanson and Lawson [9]. The most familiar formulation is due to Stewart [14]. A slightly different formulation is given below by Barlow, Yoon and Zha [2].

Let $X(t)$ have the factorization

$$
X(t)=U(t) C(t) V(t)^{T},
$$

where

$$
\begin{aligned}
& U(t) \in \mathbf{R}^{m \times n}, V(t) \in \mathbf{R}^{n \times n} \text { orthogonal, } \\
& k n-k \\
& C(t)=\begin{array}{l}
k \\
n-k
\end{array}\left(\begin{array}{cc}
L(t) & 0 \\
F(t) & G(t)
\end{array}\right), \quad L(t), G(t) \text { lower triangular }, \\
& \left\|L(t)^{-1}\right\|^{-1} \geq \epsilon \geq\|G(t)\|, \\
& \|F(t)\|_{F}\left\|L(t)^{-1}\right\|=\eta \ll 1 .
\end{aligned}
$$

The value $\epsilon$ is the tolerance or "noise level". The value $k$ is usually referred to as $\epsilon$-rank or the rank "revealed" by the ULVD. It is not the numerical rank unless $\epsilon$ is very close to machine precision times the norm of $X(t)$, and, in practice, $\epsilon$ will often be larger than that value.

Throughout the rest of the paper "rank" will denote $\epsilon$-rank.

Let $\sigma_{i}(X(t))$ denote the $i$ th singular value of $X(t)$. The conditions in (3)-(6) are sufficient to guarantee the inequality

$$
\sigma_{k}(X(t)) \geq \epsilon>\sigma_{k+1}(X(t)) .
$$

However, for computational convenience, we weaken the requirement (6) to read

$$
\left\|L(t)^{-1}\right\|^{-1} \geq \epsilon, \quad\|G(t)\|_{F} \leq \epsilon_{F} .
$$

Here $\epsilon_{F} \approx \epsilon$. Inequalities (8) are sufficient to guarantee

$$
\sigma_{k}(X(t)) \geq \epsilon, \quad\left\|\left(\sigma_{k+1}(X(t)), \ldots, \sigma_{n+1}(X(t))\right)^{T}\right\| \leq \epsilon_{F} .
$$


Updating is a process of obtaining the ULVD of (2) by using ULVD of $X(t)$. Let $U(t)$ and $V(t)$ be partitioned into

$$
\begin{aligned}
& k \quad\left(m_{0}+t\right)-k \\
& U(t)=\left(\begin{array}{ll}
U_{1}(t) & U_{2}(t)
\end{array}\right) \\
& k \quad n-k \\
& V(t)=\left(\begin{array}{ll}
V_{1}(t) & V_{2}(t)
\end{array}\right)
\end{aligned}
$$

Due to memory size and computational complexity only $L(t), U_{1}(t)$ and $V(t)$ are stored in some large scale applications [7]. Thus we need an efficient, stable update routine for these applications.

Updating algorithms given by Stewart [14], Yoon and Barlow [1, 15] and Barlow, Erbay and Zhang [3] append the new data to the bottom of the data matrix which makes them unsuitable for these applications. In this paper we present an $O\left(n^{2}\right)$ updating algorithm which appends the new data to the top of matrix. This enables us to update without storing $F(t)$ and $G(t)$ as well as $U_{2}(t)$. Numerical results show that our algorithm without storing $F(t)$ and $G(t)$ is as good as the ones that store $F(t)$ and $G(t)$.

\section{ULVD Updating Algorithm}

For the sake of notational simplicity we consider $X$ to be a matrix of order $n$. We now make notational simplifications of (3)-(7) by writing

$$
X=X(t), \quad U=U(t), \quad C=C(t), \quad V=V(t),
$$

and

$$
C=\begin{array}{cc}
k & n-k \\
n-k & \left(\begin{array}{cc}
L(t) & 0 \\
F(t) & G(t)
\end{array}\right)=\left(\begin{array}{cc}
L & 0 \\
F & G
\end{array}\right),
\end{array}
$$

for the current value of $t$. Partition $U$ and $V$ as

$$
U=\left(\begin{array}{cc}
k & \left(m_{0}+t\right)-k \\
U_{1} & U_{2}
\end{array}\right), \quad V=\left(\begin{array}{cc}
k & n-k \\
V_{1} & V_{2}
\end{array}\right) .
$$

Then

$$
X=U_{1} L V_{1}^{T}+U_{2} F V_{1}^{T}+U_{2} G V_{2}^{T}
$$

Define

$$
E=U_{2} F V_{1}^{T}+U_{2} G V_{2}^{T} .
$$

Observe that

$$
U_{1}^{T} E=0, \quad E=\left(I-U_{1} U_{1}^{T}\right) X .
$$

Before we give the steps of our algorithm, we define

$$
\mathbf{d}^{T}=\mathbf{x}^{T} V .
$$




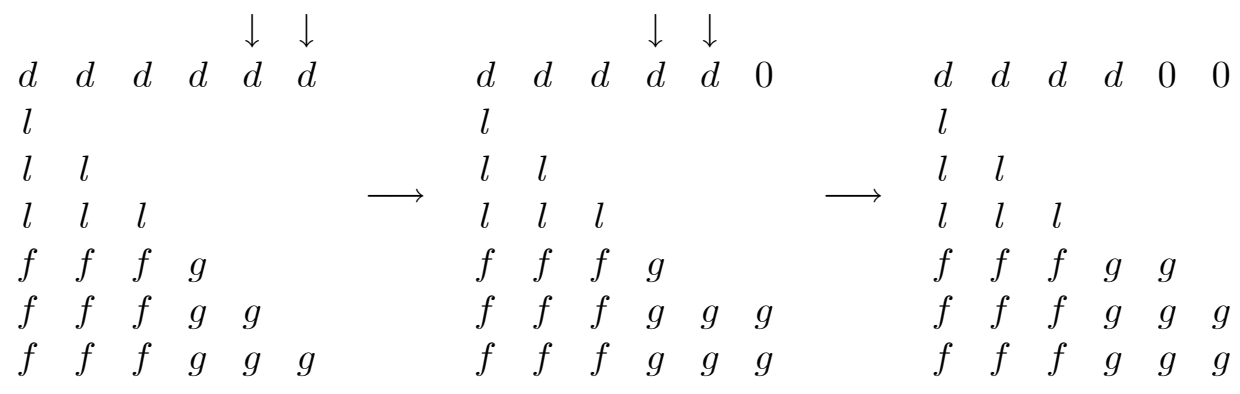

Figure 1: Reduction of the vector $\mathbf{d}_{2}$

Thus computing the ULVD of $\left(\begin{array}{c}\mathbf{x}^{T} \\ \alpha X\end{array}\right)$ reduces to updating $\left(\begin{array}{c}\mathbf{d}^{T} \\ \alpha C\end{array}\right)$.

Below are the steps of our new updating algorithm.

\section{Algorithm 1}

Step 1. Let $\mathbf{d} \in \mathbf{R}^{n \times 1}$ be partitioned

$$
\mathbf{d}=\underset{n-k}{k}\left(\begin{array}{l}
\mathbf{d}_{1} \\
\mathbf{d}_{2}
\end{array}\right)
$$

Step 2. By using Givens rotations construct an orthogonal matrix $\hat{V}_{1} \in \mathbf{R}^{(n-k) \times(n-k)}$ such that

$$
\mathbf{d}_{2}^{T} \hat{V}_{1}=\xi \mathbf{e}_{1}^{T}, \quad G^{(1)}=G \hat{V}_{1}, \quad \xi=\left\|\mathbf{d}_{2}\right\|
$$

where $G^{(1)}$ is an upper Hessenberg matrix. See Figure 1.

Step 3. Find the singular triplet $(\mathbf{u}, \boldsymbol{\sigma}, \mathbf{v})$ of the matrix,

$$
\left(\begin{array}{cc}
\mathbf{d}_{1}^{T} & \xi \\
L & 0
\end{array}\right)
$$

where $\sigma$ is the smallest singular value.

Step 4. If $(\sigma \geq \epsilon)$ then construct a $(k+1) \times(k+1)$ orthogonal matrix $\hat{V}_{2}$ via Givens rotations such that

$$
\begin{gathered}
\left(\begin{array}{c}
\mathbf{d}_{1} \\
\xi
\end{array}\right)^{T} \hat{V}_{2}=\|\mathbf{d}\| \mathbf{e}_{1}^{T}, \\
L^{(1)}=\left(\begin{array}{c}
\|\mathbf{d}\| \mathbf{e}_{1}^{T} \\
L \hat{V}_{2}(1: k, 1: k+1)
\end{array}\right),
\end{gathered}
$$




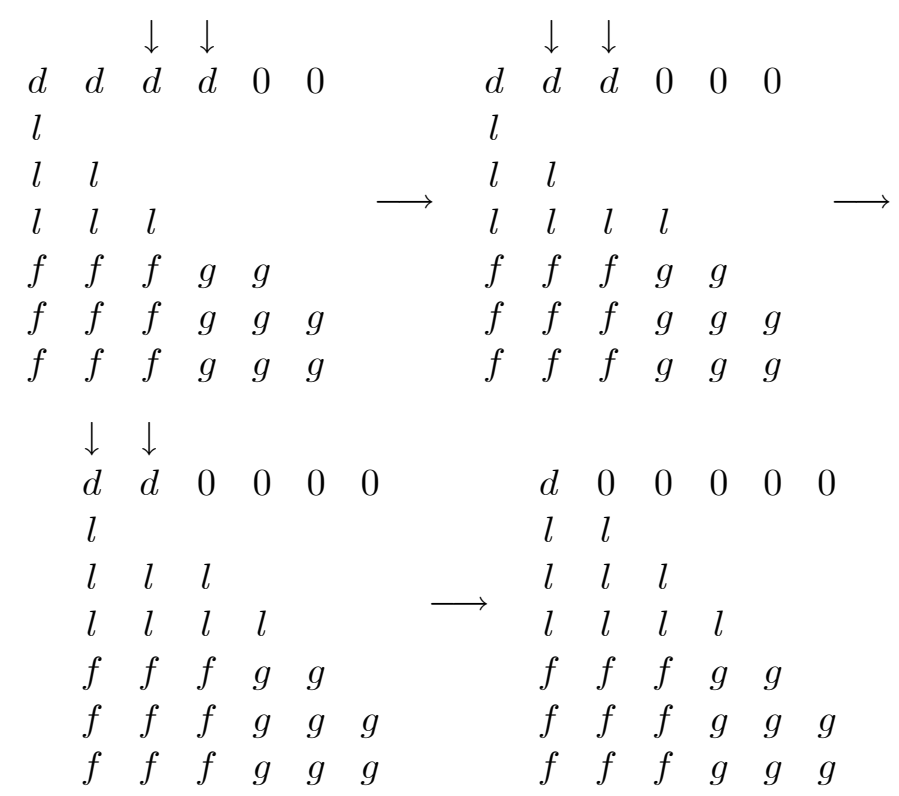

Figure 2: Reducing the vector $\mathbf{d}$.

$$
\left(F^{(1)} \quad G^{(2)}(:, 1)\right)=\left(\begin{array}{ll}
F \quad G^{(1)}(:, 1)
\end{array}\right) \hat{V}_{2}
$$

where $G^{(2)}(2: n,:)=G^{(1)}(2: n,:)$ is an upper Hessenberg matrix. See Figure 2.

else if $(\sigma<\epsilon)$ then use Givens rotations to construct $(k+1) \times(k+1)$ orthogonal matrices $\hat{V}_{2}$ and $\hat{U}_{1}$ such that

$$
\begin{gathered}
\hat{V}_{2}^{T} \mathbf{v}=\mathbf{e}_{k+1}, \\
\hat{L}=\hat{U}_{1}\left(\begin{array}{cc}
\mathbf{d}_{1}^{T} & \xi \\
L & 0
\end{array}\right) \hat{V}_{2} \quad \text { lower triangular, } \\
\left(\begin{array}{cc}
\hat{F} & \hat{g}
\end{array}\right)=\left(\begin{array}{ll}
F & G^{(1)}(:, 1)
\end{array}\right) \hat{V}_{2} .
\end{gathered}
$$

Take

$$
L^{(1)}=\hat{L}(1: k, 1: k), \quad\left(\begin{array}{cc}
F^{(1)} & G^{(2)}(:, 1)
\end{array}\right)=\left(\begin{array}{cc}
\mathbf{e}_{k+1}^{T} \hat{L} & 0 \\
\hat{F} & \hat{g}
\end{array}\right)
$$

with $G^{(2)}(2: n,:)=G^{(1)}(2: n,:)$. See Figure 3.

Step 5. By using Givens rotations construct orthogonal matrix $\hat{U} \in \mathbf{R}^{(n+1) \times(n+1)}$ such that

$$
\left(\begin{array}{cc}
L^{(2)} & 0 \\
F^{(2)} & G^{(3)} \\
0 & 0
\end{array}\right)=\hat{U}^{T}\left(\begin{array}{cc}
L^{(1)} & 0 \\
F^{(1)} & G^{(2)}
\end{array}\right) .
$$




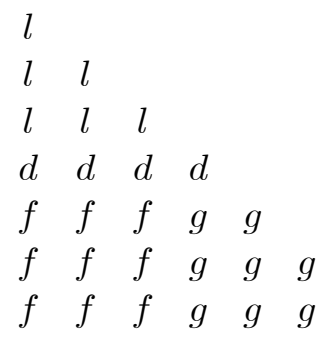

Figure 3: Moving $\mathbf{d}$ to Down as in Step 4

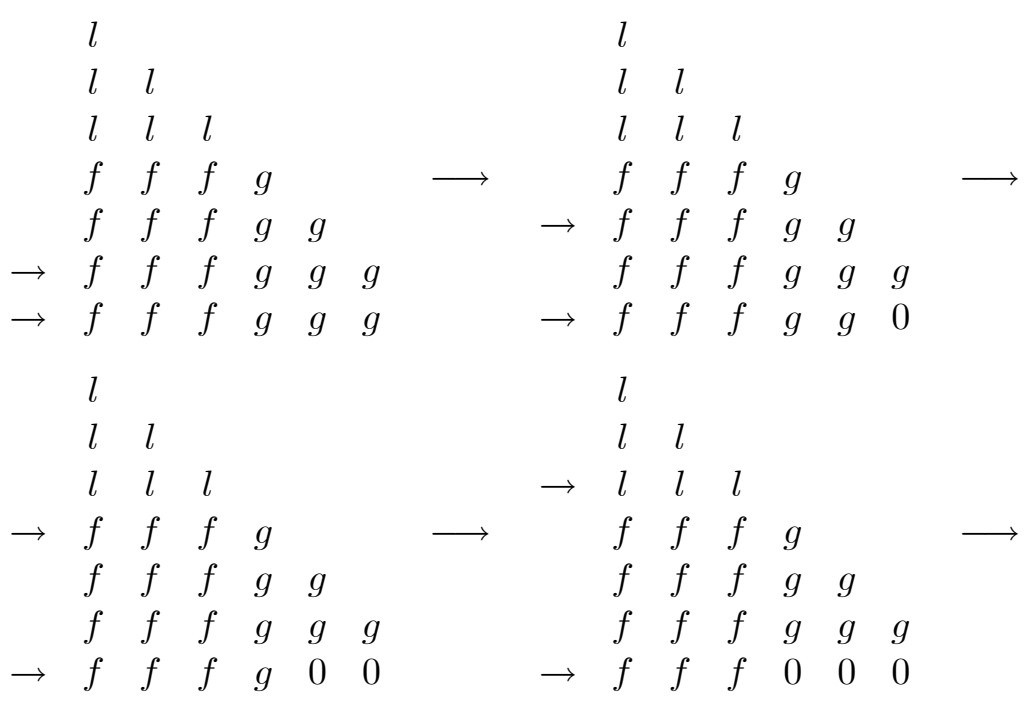

Figure 4: Eliminating the last $n-k$ of the Last Row

where $L^{(2)}$ and $G^{(3)}$ are lower triangular matrices. See Figure 4 and Figure 5. We assume that the rank of the updated matrix does not change.

Step 6. Define

$$
L(t+1)=L^{(2)} ; F(t+1)=F^{(2)} ; G(t+1)=G^{(3)} .
$$

In the environment where $F$ and $G$ are not available we skip Step 2 and define $\xi=\left\|\mathbf{d}_{2}\right\|$ as in this step. In later steps we ignore all $F$ 's and $G$ 's. However, we keep and maintain the matrix $E$ in (15).

Unlike other updating algorithms, our algorithm adds a new data vector to the top of the matrix. This has big advantages in the applications where $X$ is large and $L$ is small. To do an update no need to store both $F$ and $G$ 


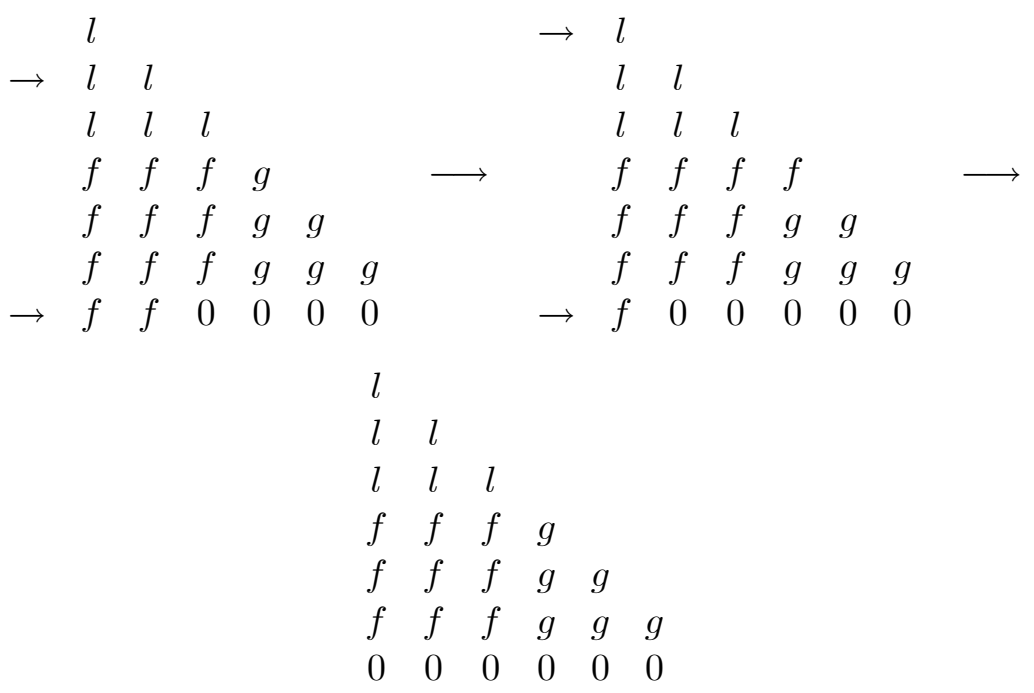

Figure 5: Eliminating the first $k$ of the Last Row

as well as $U_{2}$. Moreover, if the new data row increases the rank then it can be shown that the inequality (27) holds. In this case there is no need for any refinement procedure. Otherwise it is possible for the matrix $L$ to lose its rank revealing structure, in which case the refinement procedure [4] will restore it.

The estimation of the smallest singular value requires $\lambda k^{2}$ flops, where $\lambda$ is a small constant. This can be achieved by using any of a number reliable condition estimators such as a few Lanczos or power iterations $[6,10,11]$. When the operations are counted, the overall complexity of the algorithm can be found $O\left(n^{2}\right)$ flops.

Our numerical results show that the algorithm without storing $F$ and $G$ is as good as the ones that store $F$ and $G$.

Proposition 2 Consider the terminology of the updating algorithm 1. Assume that $\operatorname{rank}(X(t+1))>\operatorname{rank}(X(t))$ for some time $t$. Then

$$
\left\|\left(\begin{array}{lll}
F(t+1) & G(t+1)
\end{array}\right)\right\|_{F} \leq\left\|\left(\begin{array}{cc}
F(t) & G(t)
\end{array}\right)\right\|_{F} .
$$

Proof. In step 5 of our algorithm we eliminate the extra row by Givens rotations. Let $Q=Q_{k} Q_{k+1} \ldots Q_{n}$ be the product of rotations that eliminates last $n-k$ elements of the vector $\left(F^{(1)} G^{(2)}\right)^{T} \mathbf{e}_{n-k}$ as in Figure 4. Then define

$$
\left(\begin{array}{cc}
\bar{F} & \bar{G}
\end{array}\right)^{T} \mathbf{e}_{n+1}=\left(\begin{array}{cc}
F^{(1)} & G^{(2)}
\end{array}\right)^{T} \mathbf{e}_{n+1} Q^{T}
$$

After this process we eliminate first $k$ elements of the vector $\left(\begin{array}{ll}\bar{F} \quad \bar{G}\end{array}\right)^{T} \mathbf{e}_{n-k}$ to obtain $\left(\begin{array}{cc}F^{(2)} & G^{(3)}\end{array}\right)$. We observe that

$$
\left\|\left(F^{(2)} G^{(3)}\right)\right\|_{F}^{2}=\left\|\left(\begin{array}{cc}
F^{(1)} & G^{(2)}
\end{array}\right)\right\|_{F}^{2}-\left\|\bar{F}^{T} \mathbf{e}_{n+1}\right\|^{2}
$$


The proof follows from the fact that $\left\|\bar{F}^{T} \mathbf{e}_{n+1}\right\|_{2} \geq 0$.

Corollary 3 Assume the hypothesis and terminology of proposition 2. Then

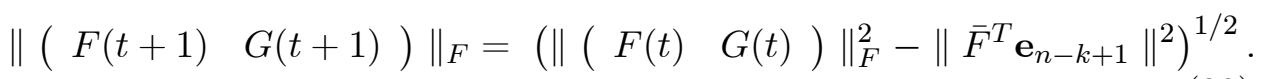

Proof. Due to orthogonal invariance of Frobenius norm we have that

$$
\begin{gathered}
\left\|\left(\begin{array}{cc}
F & G^{(1)}
\end{array}\right)\right\|_{F}=\left\|\left(\begin{array}{cc}
F & G
\end{array}\right)\right\|_{F} \\
\left\|\left(\begin{array}{cc}
F^{(1)} & G^{(2)}
\end{array}\right)\right\|_{F}=\left\|\left(\begin{array}{cc}
F & G^{(1)}
\end{array}\right)\right\|_{F} .
\end{gathered}
$$

Thus, the equality (30) follows from the equality (29).

Proposition 4 Consider the terminology of the updating algorithm 1. Assume that $\operatorname{rank}(X(t+1))=\operatorname{rank}(X(t))$ for some time $t$. Then

$\left.\left\|\left(\begin{array}{ll}F(t+1) & G(t+1)\end{array}\right)\right\|_{F}=\left(\begin{array}{ll}(F(t) & G(t)\end{array}\right)\left\|_{F}^{2}+\right\| \mathbf{x}\left\|^{2}-\right\| \bar{F}^{T} \mathbf{e}_{n-k} \|^{2}\right)^{1 / 2}$

where $\bar{F}$ is as in Proposition 2.

Proof. We observe that

$$
\left\|\left(\begin{array}{ll}
F^{(1)} & G^{(2)}
\end{array}\right)\right\|_{F}^{2}=\left\|\left(\begin{array}{cc}
F & G
\end{array}\right)\right\|_{F}^{2}+\|\mathbf{x}\|^{2} .
$$

Then proof is immediate from (29).

We observe that the former two propositions above suggest that the Frobenius norm of the matrix $(F(t+1) \quad G(t+1))$ can be tracked using $O(n)$ flops at each updating step. We also observe that these two propositions and the corollary can easily be modified to calculate the Frobenius norm of the matrix $(F(t+1) \quad G(t+1))$ of the sliding window problem.

\section{Issues On The Sliding Window Method}

Consider the sliding window problem. The Cauchy's interlace theorem on rank-one modification [12, pp. 202-204] tells us that if a matrix $X(t)$ has $k$ singular values greater than $\epsilon$ then the new matrix $X(t+1)$ that obtained by adding a new data $\mathbf{x}(t+1)$ to $X(t)$ has either $k$ or $k+1$ singular values greater than $\epsilon$. However, the forgetting factor $\alpha$ in the sliding window problem may cause ,in addition to above possibilities, less than $k$ singular values greater than $\epsilon$. To handle the former possibility we deflate the resulting triangular matrix $L(t+1)$ repeatedly until $L(t+1)$ has no singular values less than $\epsilon$. 


\section{Some Computational Examples}

This section presents two examples from our numerical experiments. These tests were performed using Matlab on a Ultra5 SUN station in IEEE Standard double precision with machine epsilon $2^{-53} \approx 1.102 \times 10^{-16}$. We use exponential window technique to test our algorithm.

The ULVD of the initial window matrix $X(0)$, which consists of the first $m_{0}$ rows of $X$, can be obtained by computing its QL factorization

$$
X(0)=U(0)\left(\begin{array}{c}
C(0) \\
0
\end{array}\right) \text {, }
$$

where $U(0)$ is an $m_{0} \times m_{0}$ orthogonal matrix and $C(0)$ is an $n \times n$ lower triangular, followed by computing the ULVD of $C(0)$. This involves two steps: estimating an approximate left singular vector $\mathbf{u}_{n}$ of unit norm of $C(0)$ which corresponds to the smallest singular value $\sigma_{n}$ of $C(0)$, and computing an orthogonal matrix $\bar{U}(0)$ such that $\mathbf{u}_{n}^{T} \bar{U}(0)=\mathbf{e}_{n}^{T}$ and orthogonal matrix $\bar{V}(0)$, so that

$$
\sigma_{n}=\left\|\mathbf{u}_{n}^{T} C(0)\right\|=\left\|\left(\mathbf{u}_{n}^{T} \bar{U}(0)\right)\left(\bar{U}(0)^{T} C(0) \bar{V}(0)\right)\right\|=\left\|\mathbf{e}_{n}^{T}\left(\bar{U}(0)^{T} C(0) \bar{V}(0)\right)\right\|,
$$

which is the size of the last row of $C(0)$. Here, $\bar{V}(0)$ is applied to restore $\bar{U}(0)^{T} C(0)$ into the lower triangular form. We repeat this deflation process until all of the small rows of $C(0)$ appear in the decomposition yielding the ULVD of $X(0)$.

We compare our algorithm with the algorithm given by Barlow, Erbay and Zhang [3] by means of MATLAB's SVD algorithm. From now on we will refer to Barlow, Erbay and Zhang's algorithm as the BEZ algorithm.

Let

$$
X(t)=W(t) \Sigma(t) Y(t)^{T}, \quad Y(t)=\left(\begin{array}{ll}
Y_{1}(t) & Y_{2}(t)
\end{array}\right)
$$

be the SVD of $X(t)$ obtained by the Matlab's SVD algorithm at step $t$. Also, let

$$
X(t)=U(t) C(t) V(t)^{T}, \quad V(t)=\left(\begin{array}{ll}
V_{1}(t) & V_{2}(t)
\end{array}\right)
$$

be the ULVD of $X(t)$ obtained by our algorithm. Finally, let

$$
X(t)=\bar{U}(t) \bar{C}(t) \bar{V}(t)^{T}, \quad \bar{V}(t)=\left(\begin{array}{ll}
\bar{V}_{1}(t) & \bar{V}_{2}(t)
\end{array}\right)
$$

be the ULVD of $A(t)$ computed by the BEZ algorithm. Define the angles between the subspaces

$$
\sin \theta_{1}(t)=\left\|Y_{1}^{T}(t) V_{2}(t)\right\| ; \quad \sin \theta_{2}(t)=\left\|Y_{1}^{T}(t) \bar{V}_{2}(t)\right\|
$$

The angles $\theta_{i}, i=1,2$ represent, respectively, error between the true noise subspace from the Matlab's SVD and the approximate error from the ULVD by our algorithm, the approximate error from the ULVD by the BEZ algorithm. We plotted $\sin \theta_{i}$ for $i=1,2$ on the $\log 10$ scale to have better view of errors in solid and dotted lines, respectively. 

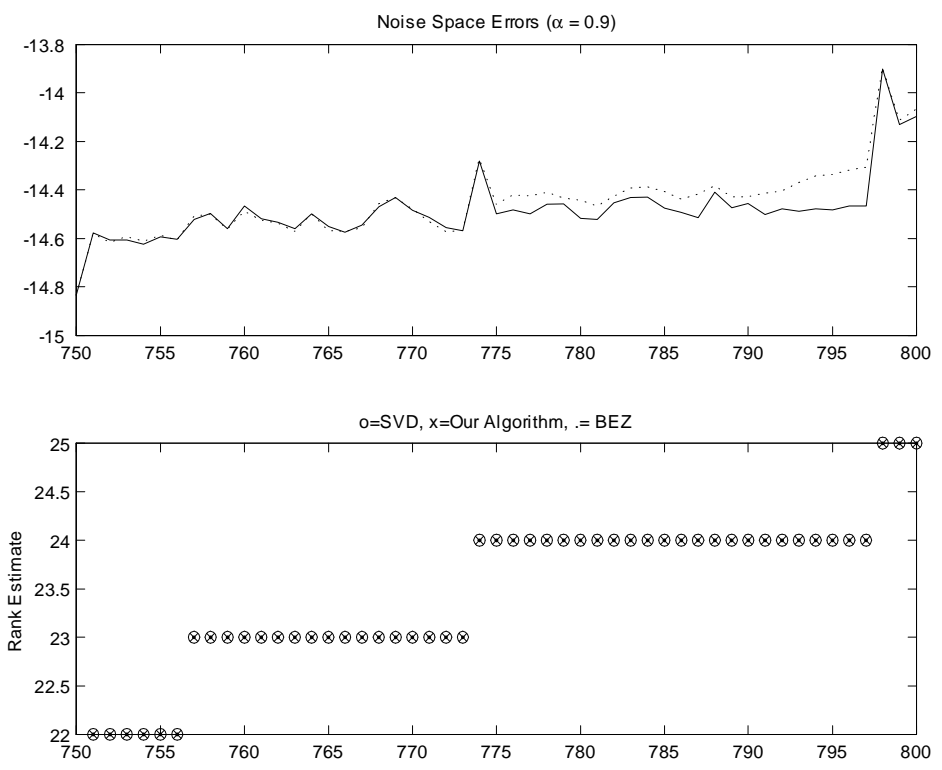

Figure 6: The plots show some results obtained by example 5

Example $5 X$, 800-by-75 random matrix, chosen from a uniform distribution on the interval $(0,1) .775$ randomly chosen rows of $X$ multiplied by $\eta=10^{-9}$ in order to vary the rank of the matrix, and $\epsilon \approx 10^{-8}$. The initial matrix $X(0)$ is of size 750-by-75 and the forgetting factor $\alpha=0.9$.

Example $6 X$, 800-by-75 random matrix, chosen from a uniform distribution on the interval $(0,1) .775$ randomly chosen rows of $X$ multiplied by $\eta=10^{-9}$ in order to vary the rank of the matrix, and $\epsilon \approx 10^{-8}$. The initial matrix $X(0)$ is of size 750-by-75 and the forgetting factor $\alpha=0.8$.

The first plot in Figures (6) and (7) show the noise subspace errors obtained by our algorithm (solid line) and the BEZ algorithm (dotted line). Thus, by the quantities in (35) our algorithm is slightly better than BEZ algorithm.

The second plot in Figures (6) and (7) show the rank estimates by our algorithm, the BEZ algorithm and MATLAB's SVD algorithm. The rank estimate of these algorithms are identical in spite of frequent rank changes. The horizontal axes represent the window steps.

The table (1) shows the overall CPU time in seconds of each algorithm during the sliding window process for each example. 


\begin{tabular}{|c|c|c|c|}
\hline & Our algorithm & BEZ algorithm & SVD \\
\hline By example 5 & 2.87 & 10.73 & 130.59 \\
\hline By example 6 & 2.69 & 10.30 & 129.32 \\
\hline
\end{tabular}

Table 1: Overall CPU time in seconds of the algorithms
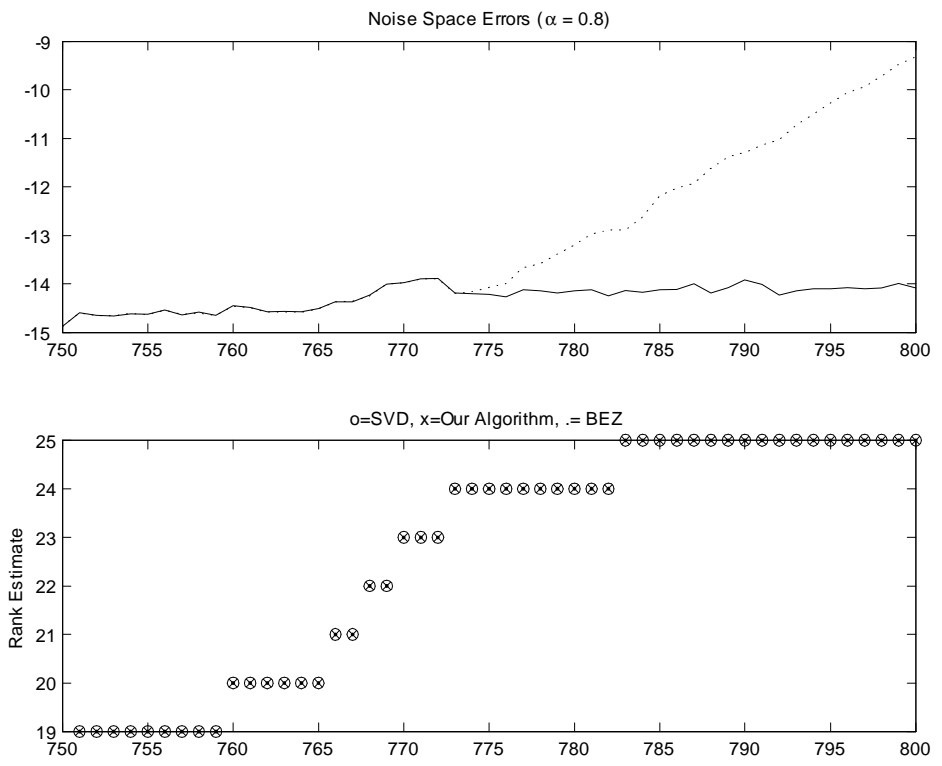

Figure 7: The plots show some results obtained by example 6

\section{Conclusion}

We have presented an updating algorithm which is suitable for large scaled problems in which the rank of a matrix is small compared to the its size. Our numerical results show that the updating algorithm is promising.

\section{References}

[1] J.L. Barlow and P.A. Yoon, Solving Recursive TLS Problems Using Rank-Revaling ULV Decomposition, In S. Van Huffel, editor, Proc. Workshop on TLS and Errors-In-Variables, Philadelphia, PA, SIAM Publications, 1997, pp. 117-126.

[2] J.L. Barlow P.A. Yoon and H. Zha, An Algorithm And A Stability Theory For Downdating The ULV Decomposition, BIT, 36(1996), 15-40.

[3] J.L. Barlow H. Erbay and Z. Zhang, A modified Gram-Schmidt based downdating technique for the ULV decompositions with applications to 
recursive TLS problems, Adv. Sign. Proc. Alg., Arch. and Impl. IX, F.T. Luk, Editor, SPIE Proc., Bellingham, WA, 3807(1999), 247-257.

[4] H. Erbay and J.L. Barlow, Recursive ULV decompositions and an alternative refinement algorithm, Adv. Sign. Proc. Alg., Arch. and Impl. X, F.T. Luk, Editor, SPIE Proc., Bellingham, WA, 4116(2000), 157-166.

[5] D.K. Faddeev V.N. Kublanovskaya and V.N. Faddeeva, Solution Of Linear Algebraic Systems With Rectangular Matrices, Proc. Steklov Inst. Math., 96(1968), pp. 93-111.

[6] R. Ferng G.H. Golub and R.J. Plemmons, Adaptive Lanczos Methods for Recursive Condition Estimation, Numerical Algorithms, 1(1991), pp. 1-20.

[7] R. Fierro L. Vanhamme and S. Van Huffel, Total Least Squares Algorithms Based On Rank-Revealing Complete Orthogonal Decompositions, SIAM Publications, 1997, pp. 99-116.

[8] M. Gu and S. Eisenstat, Downdating The Singular Value Decomposition, SIAM J. Matrix Anal. Appl., 16(1995), pp. 793-810.

[9] R.J. Hanson and C.L. Lawson, Extensions And Applications Of The Householder Algorithm For Solving Linear Least Squares Problems, Math. Comp., 23(1969), pp. 787-812.

[10] N.J. Higham, A Survey of Condition Number Estimation for Triangular Matrices, SIAM Review, 29(1987), pp. 575-596.

[11] N.J. Higham, Experience with a Matrix Norm Estimator, SIAM J. Stat. Comp., 11(1990), pp. 804-809.

[12] B.N. Parlett, The Symmetric Eigenvalue Problem, SIAM Publications, 1997.

[13] G.W. Stewart, Error Analysis of QR Updating With Exponential Windiwing, Mathematics of Computations, 59(1992), pp. 135-140.

[14] G.W. Stewart, Updating A Rank-Revealing ULV Decomposition, SIAM J. Matrix Anal. Appl., 14(1993), pp. 494-499.

[15] P.A. Yoon and J.L. Barlow, An Efficient Rank Detection Procedure For Modifying The ULV Decomposition, BIT, 38(1998), pp. 781-801.

Hasan Erbay

Kırıkkale University

Department of Mathematics 
Yahşihan

Kirıkkale , 71100

TURKEY

hxe68@yahoo.com

Jesse L. Barlow

The Pennsylvania State University

Department of Computer Science and Engineering

Information Science and Technology Building

University Park

Pennsylvania , 16802-6882

USA

barlow@cse.psu.edu 